\title{
The Development of Internal Representations of Magnitude and Their Association with Arabic Numerals
}

\author{
Orly Rubinsten, Avishai Henik, Andrea Berger, and Sharon Shahar-Shalev
}

Ben-Gurion University of the Negev, Beer-Sheva, Israel

\begin{abstract}
Developmental aspects of number concepts were evaluated using participants from the beginning and end of first grade (6-7 years old), third and fifth grades (7-11 years old), and university (22 years old). Participants evaluated the numerical value or physical size of stimuli varying along both dimensions. The numerical distance effect appeared in all groups. In contrast, the size congruity effect started to appear only at the end of first grade. Based on our results, a model of internal representation of magnitude claiming that there are two different representations was propose. At the beginning of first grade children can automatically access only one of these representations and only from the end of first grade can they access both of these representations. ๑ 2002 Elsevier Science
\end{abstract}

Key Words: Arabic numeral; quantity; automaticity; development.

Moyer and Landauer (1967) were the first to find that the larger the numerical difference between two digits, the shorter the time required to decide which is larger. For example, it takes longer to decide that " 8 " is larger than " 6 " than to decide that " 8 " is larger than " 1 ." This distance effect has since been reported in numerous studies (e.g., Banks, Mermelstein, \& Yu, 1982; Dehaene, 1989; Dehaene, Dupoux, \& Mehler, 1990; Duncan \& McFarland, 1980; Henik \& Tzelgov, 1982; Link, 1990; Moyer, 1973; Moyer \& Bayer, 1976; Parkman, 1971; Schwarz \& Heinze, 1998; Tzelgov, Meyer, \& Henik, 1992). Dehaene (1997) trained university students to avoid the distance effect and found that even after 1600 training trials, participants were still slower with close digits than with more distant ones. Moyer and Landauer (1967) suggested that people convert written or auditory numbers into analog magnitudes. The comparison between these magnitudes is made in much the same way that comparisons are made between physical stimuli such as lengths of lines. It has been postulated that the source of the distance effect is the overlap between representations of numbers. That is, the

This work was supported by a grant to A. Henik from the Israel Science Foundation founded by the Israel Academy of Sciences and Humanities. We thank Mrs. Kineret Hadad, principal of Oriyan Elementary School in Beer-Sheva, Israel, for allowing us to conduct our research in the school. We also thank the teachers and students in Oriyan Elementary School for helping and participating in the experiment.

Address correspondence and reprint requests to Avishai Henik, Department of Behavioral Sciences, Ben-Gurion University of the Negev, Beer-Sheva, Israel. Fax: 972-8-6472932. E-mail: henik@bgumail.bgu.ac.il. 
internal semantic representations of close numbers, such as " 1 " and " 2 ," overlap more than those of more distant numbers (Dehaene \& Changeux, 1993; Gallistel $\&$ Gelman, 1992). Several investigators argue that numerals or semantic sizes are directly represented in a linear order (Rabinowits, Grant, Howe, \& Walsh, 1994; Tzelgov, Yehene, Kotler, \& Alon, 2000). In addition, it is suggested that not only do numbers map onto internal magnitudes or quantitative values, but also that this mapping is automatic (Dehaene, 1992; Dehaene \& Akhavein, 1995; Henik \& Tzelgov, 1982; Tzelgov, Meyer, \& Henik, 1992; Zbrodoff \& Logan, 1986).

What is automaticity? Posner (1978) likened automaticity to reflexive behavior and suggested three indicators for such activity: automatic processes occur without intention and without conscious awareness, and they can run in parallel with other cognitive processes since they do not require attention in order to be executed (see also Hasher \& Zacks, 1979). However, these classic requirements have been criticized during the past decades (e.g., Carr, 1992; Logan, 1985). It has been shown that automatic processes are sensitive to attentional requirements and expectations (Zbrodoff \& Logan, 1986). Tzelgov, Henik, Sneg, and Baruch (1996) suggested that a process is automatic if it does not need monitoring to be executed. That is, once the process is triggered, either by intention or by external unintentional stimulation, it runs by itself without the need of conscious monitoring.

Several researchers argued that the distance effect indicates automatic processing. For example, Dehaene and Akhavein (1995) argued that the distance effect indicates that the representation of number magnitude is automatically accessed whether or not the task requires the use of numerical values. The participants in their experiment had to decide whether two digits presented together (e.g., 3-3 or three-3) were identical. The distance effect was obtained in most trials. Another effect that indicates automaticity of processing the numerical value of digits appears in a Stroop-like paradigm. In this paradigm participants are shown pairs of Arabic numerals and asked to decide which of the two numerals is larger. Participants are asked to relate to the physical size of the digits and to ignore the numerical value, or they can be asked to relate to the numerical value of the digits and ignore their physical size. The two digits can be incongruent, congruent, or neutral. In the congruent trials the numerically larger numeral is also physically larger (e.g., 5 2), in the incongruent trials the numerically larger numeral is physically smaller (e.g., 5 2), and in the neutral trials only the physical size is different and the digits have the same numerical value or the other way around (e.g., 55 or 52 , for physical comparisons and numerical comparisons, respectively). The size congruity effect (Paivio, 1975), an indication for automatic processing of the irrelevant dimension, is manifested by shorter reaction times (RT) for congruent trials than for incongruent trials (Besner \& Coltheart, 1979; Dehaene, 1992; Henik \& Tzelgov, 1982; Schwarz \& Heinze, 1998; Tzelgov, Meyer, \& Henik, 1992). In general, the outcome of this Stroop-like paradigm suggests an automatic activation of numerical information. That is, when participants judge the physical sizes of digits they cannot ignore their numerical values. 
Interestingly, the numerical distance between two digits influences performance only in numerical judgments and is absent in physical judgments (Tzelgov, Meyer, \& Henik, 1992). In contrast, the size congruity effect is found in both numerical and physical judgments (Henik \& Tzelgov, 1982; Tzelgov, Meyer, \& Henik, 1992). We should mention here that Dehaene and Akhavein (1995) found a distance effect even when the numerical value (semantic information) was irrelevant to their task. We believe that this finding does not necessarily contradict the common lack of distance effect in physical judgments in the Stroop-like paradigm. Dehaene and Akhavein asked their participants to decide if two numbers (Arabic numerals or written words) were identical (in their form) or not. Physical sizes were roughly the same. There were several differences between the same-different task employed by Dehaene and Akhavein and the Stroop-like paradigm used in comparative judgements. These differences might have produced the different results. For example, Boucart and Humphteys (1994) have recently suggested that processing of an irrelevant dimension is contingent upon the saliency of the relevant dimension. It is possible that global shape is less salient and requires elaborate processing, which in turn entails processing of other dimensions of the stimuli, like the numerical value. In contrast, physical comparisons involve a salient dimension, which in turn prevents semantic processing of the stimuli (e.g., numerical dimension).

One can distinguish between two modes of automatic processing. When a process is part of the task requirements it is intentional, whereas when it is not part of the task requirements (but nevertheless affects performance) it is autonomous (Tzelgov et al., 1996). Hence, the distance effect might be considered a product of intentional automatic processing and the size congruity effect might be considered a product of autonomous automatic processing.

\section{EARLY NUMERICAL DEVELOPMENT}

The ability to automatically process the quantitative values of Arabic numerals may play an important role in the acquisition and implementation of skilled calculation (Griffin, Case, \& Capodilupo, 1995). Hence, studying this ability has important implications for theories of education and child development in the field of number processing. Accordingly, the current study examines developmental changes in the representations of magnitude and to what extent these representations are activated automatically.

It may be suggested that by the time children associate Arabic numerals with symbolic values they already exhibit magnitude information that is independent of formal school instruction. Piaget (1953) argued that "It is a great mistake to suppose that a child acquires the notion of numbers and other mathematical concepts just from teaching. On the contrary, to a remarkable degree he develops them himself independently and spontaneously" (p. 74; see also Piaget, 1952). However, he believed that children are born without any preconceived idea about arithmetic. Only during the first 8 years, while manipulating collections of objects in the environment, do they discover the true meaning of numbers. 
Contrary to Piaget's theory, there is some recent evidence that shows that conceptual understanding of counting is present very early in life. Dehaene (1997) summarized his book by saying that ". . . in children, numerical estimation, comparison, counting, simple addition and abstraction all emerge spontaneously without much explicit instruction" (p. 245).

Gallistel and Gelman (1992) and Gelman (1978) suggested that preschool-age children possess a range of sophisticated quantitative abilities reflecting number abstraction principles. Most studies in the field relate to this early knowledge as "preverbal counting" (Gallistel \& Gelman, 1992; Whalen, Gallistel, \& Gelman, 1999), internal representation of magnitude (Gallistel \& Gelman, 1992; Ta'ir, Bresner, \& Ariel, 1997), or implicit knowledge (Gelman, 1982, 1994). Moreover, this implicit knowledge is evident in their behavior even if they cannot express it explicitly. That is, this knowledge is activated automatically. An important question is whether this implicit knowledge is activated when it is irrelevant to the task at hand. For example, will an irrelevant numerical value be activated in young children so that it interferes with a relevant physical comparison?

Only a few studies tested the distance effect in children. Duncan and McFarland (1980) asked children to decide whether two Arabic numerals were identical. This task required a simple visual matching and yet the authors found a distance effect in participants from kindergarten, first grade, third grade, and fifth grade. The authors argued that their findings suggest that children as young as 6 years old access magnitude information automatically. Temple and Posner (1998) found a distance effect in 5- and 9-year-old children. Sekular and Mierkiewicz (1977) found that the function relating judgment time to numerical difference is steeper for children in kindergarten and first grade relative to children in fourth and seventh grades. Huntley-Fenner and Cannon (2000) found that 3 to 5-year-olds find it more difficult to discriminate arrays in a 2:3 relationship than arrays in a 1:2 relationship. Even infants show the distance effect: Xu and Spelke (2000) found that infants could discriminate 8 versus 16 items, but not 8 versus 12 items. Note that the distance effect indicates only what was defined previously as intentional automatic processing (Tzelgov, Henik, Sneg, \& Baruch, 1996). A more stringent test of the automaticity of numerical processing (or autonomous automatic processing according to the same categorization) is to find if numerical values interfere with processing when they are completely irrelevant to the task at hand. One possible sign of this would be the size congruity effect of numerical values found in the Strooplike paradigm. Girelli, Lucangeli, and Butterworth (2000) (see also Butterworth, 1999) did not find a size congruity effect in the performance of first-grade children when the task was to compare the physical sizes of Arabic numerals and to ignore their numerical values. The size congruity effect emerged only in the third grade and was also significant in the fifth grade. They argued that their findings indicate that children as young as 6 years old do not automatically access the quantitative values of Arabic numerals since numerical values did not interfere with physical judgments. Note that there is another 
measure of automaticity that was not discussed in their work, which is reflected in the distance effect (Dehaene \& Akhavein, 1995). It should be noted that Girelli, Lucangeli, and Butterworth (2000) examined the effect of laterality (and not distance) by comparing bilateral pairs (i.e., one digit was smaller and the other larger than 5) and unilateral pairs (i.e., both digits either smaller or larger than 5). For the bilateral stimuli they employed pairs with distance 5 (e.g., 16 ), while for the unilateral stimuli they employed pairs with distance 1 (e.g., 12 ). The authors did report a significant effect of laterality (i.e., RTs to bilateral pairs were shorter than RTs to unilateral pairs) in numerical comparisons regardless of age, which could indicate a distance effect. However, their experimental arrangement confounded laterality and distance, so the fact that bilateral pairs were responded to faster than unilateral pairs could have been due either to the influence of laterality or to the distance effect. Hence, their findings still cannot provide us with a very specific idea of how magnitudes are internally represented in adults as well as in children.

It should be mentioned here that the Girelli, Lucangeli, and Butterworth (2000) article was published when we had just finished running our experiment and, at that time, we were unaware of its existence.

\section{HOW CAN DISTANCE AND SIZE CONGRUITY EFFECTS CONTRIBUTE TO THE UNDERSTANDING OF THE INTERNAL REPRESENTATION OF MAGNITUDE?}

As mentioned, the distance effect indicates automatic access of numerical values (Dehaene \& Akhavein, 1995). Accordingly, one can ask, why does not it interfere with physical size comparisons? There are two alternative answers to this question. The first one suggests that these two effects (i.e., the size congruity effect and the distance effect) are dissociable because they indicate access to two different representations of internal numerical magnitude. The second suggests that these two effects represent two stages in accessing one internal representation of the number line (for an example see, Banks, 1977). In what follows we discuss these two hypotheses.

According to the two representations model, size congruity and distance effects reflect accessing two representations of numerical magnitude that are qualitatively different. Theories of skill development assume that acquisition of a cognitive skill starts by applying a mental algorithm: a set of mental computations aimed at solving the problem in question. During practice performance improves and becomes automatic. The literature suggests two mechanisms that underlie the improvement with practice. Logan (1988) proposed that with practice we accumulate instances that can be later accessed easily. Automaticity reflects direct retrieval of these instances from memory. Others assume that practice gradually improves the application of the algorithm. Note that this is the same algorithm used by the novice to solve the problem in question (Anderson, 1993). Tzelgov et al. (1996) proposed that automaticity might reflect either one of these mechanisms: memory retrieval or algorithmic processing. 
Accordingly, it is possible to propose that the distance effect and the size congruity effect reflect these two different mechanisms. The distance effect reflects an algorithmic process used to retrieve the required information from the number line. Dehaene and his colleagues (Dehaene, 1989; Dehaene, Depoux, \& Mehler, 1990) described such an algorithm. They suggested that digits are first mapped into quantities on an analogical number line. The comparison between the digits is conducted by a look-up process that probes the quantities on the number line. The variable that plays a major role in this algorithm is the analogical distance among the translated quantities, the further the quantities are located on the number line the easier it is to decide which one is larger. In contrast, the size congruity effect reflects retrieval from memory of accumulated instances involving comparisons of two numbers. According to Logan's instance theory, performance reflects direct retrieval of instances of the many stimulusspecific traces stored during acquisition. We can assume that instances of physical comparisons and of internal representations of magnitude begin to accumulate from early infancy (Gallistel \& Gelman, 1992; Siegler, 1986; Sophian, 1998; Sophian, Garyantes, \& Chang, 1997; Strauss \& Curtis, 1981; Wynn, 1992, 1995; Yonas, Granrud, \& Pettersen, 1985). However, instances of comparisons between numerical values of Arabic digits begin to accumulate later, only when these digits are studied. With practice we accumulate instances where Arabic digits indicate large or small quantities (e.g., in the pair 1-2, 2 is larger and in the pair 6-4, 6 is larger) and these can be retrieved later from memory. Note that this model (i.e., the two representations model) suggests that extracting an analogical distance and a gross estimation of numerical values (large vs small) are two different processes that involve two different mechanisms. The fact that there is a size congruity effect and no distance effect when numerical values are irrelevant (Henik \& Tzelgov, 1982) can be explained by Logan's (1988) assumption that in the race between memory-retrieval and algorithm-based processes, the former usually wins. That is, both mechanisms may be applied to the irrelevant (numerical) dimension but only the instance-based information (i.e., large/small quantities) will have an effect and will be processed in an autonomous automatic fashion. With respect to numerical comparisons, the requirement to attend to the numerical dimension entices participants to use the algorithmic process to retrieve the required information from the number line (Dehaene, 1989; Dehaene, Depoux, \& Mehler, 1990), thus creating the distance effect. This fits the results recently found in a training study by Tzelgov et al. (2000) and the notion that the algorithmic process is done in an intentional automatic fashion. Tzelgov et al. (2000) trained participants to perform magnitude judgments on Gibson figures. Even the group of participants who were trained not only with adjacent pairs but also with instances of nonadjacent pairs showed a distance effect in semantic (numerical) comparisons and a size congruity effect in physical comparisons. Note that the two representations model suggests an asymmetry between size congruity and distance effects: While one may expect a distance effect with no size congruity, the opposite is not expected. 
In contrast, the unitary representation model suggests that size congruity and distance effects reflect two stages in the process of accessing the single internal representation of the number line (e.g., Banks, 1977). The decision regarding which digit is larger is done in two steps: the first one is faster and the second is much slower. In the first and fast stage, participants decide in a very crude and general way which numeral is "large" or "small." When the two numbers are close together, so that the first step does not distinguish between them (e.g., the two have the "large" tag), another process is applied producing a more refined result. In order to get a size congruity effect there is no need to activate the refined process. The crude and fast process, that produces the "large" or "small" classification, is enough to yield this effect. This model suggests an asymmetry between size congruity and distance effects: While one may expect a size congruity with no distance effect, it is impossible to find a distance effect with no size congruity effect.

Note that the two models suggest asymmetries between size congruity and distance effects, but these asymmetries are in opposite directions. Moreover, in adults we always find both effects (a distance effect in numerical judgments and a size congruity effect in physical judgments). Hence, it is impossible to tease these two models apart by the data reported in the literature so far. It should be possible to test these two models by examining young children in early stages of number skills development.

\section{THE CURRENT STUDY}

We designed a study to look at both size congruity and distance effects. In order to examine the distance effect we used three numerical distances of 1 (e.g., 2 3), 2 (e.g., 2 4), and 4 (e.g., 2 6). Moreover, numerical distance was manipulated independently of the congruence between the physical and the numerical dimensions of the stimuli. This let us examine the development of these two aspects of automaticity in young children. In addition, we used two groups of first-grade children: those at the beginning of grade one and those at the end of this grade. This let us look more closely at this age group, where we expected to find major changes due to both maturation and schooling.

We asked our participants, ages 6 to 23 years old, to compare numerical stimuli. The two digits displayed varied along both numerical and physical dimensions. The participants evaluated (in different blocks) the physical size or the numerical value of the digits.

We also looked at the interference and facilitatory components of the size congruity effect by using neutral conditions. One can compare the neutral stimuli to the incongruent stimuli in order to examine the interference component, and one can compare the neutral stimuli with the congruent stimuli in order to examine the facilitatory component. We wanted to examine these aspects of the effects because Posner (1978) suggested that facilitation is an indicator of automaticity while interference might reflect more attentional processing. 


\section{Method}

Participants. Five groups, with 16 participants each, were examined: (1) beginning of first grade students (mean age 6.25, $S D=0.43$; 9 females and 7 males; medium socioeconomic status), (2) end of first grade students (mean age 7.32, SD $=0.43$; 9 females and 7 males; medium socioeconomic status), (3) third grade students (mean age 8.82, $S D=0.52 ; 8$ females and 8 males; medium socioeconomic status), (4) fifth grade students (mean age 11, $S D=0.5 ; 10$ females and 6 males; medium socioeconomic status), and (5) university students (mean age 22.8, $S D=2.4 ; 12$ females and 4 males; medium socioeconomic status). All participants had intact or corrected vision. The first four groups were selected from the same school. According to the school psychologist, all children had a normal IQ and none of them had any attention or memory problems. The fifth group was composed of students from Ben-Gurion University of the Negev who participated in the experiment in partial fulfillment of a course requirement.

Stimuli. A computer display stimulus consisted of two digits that appeared centered around a temporary fixation point. The center-to-center distance between the two digits was $10 \mathrm{~mm}$. Each participant performed two kinds of comparisons. In one, the relevant dimension was physical size, and in the other, numerical value. In every block there were 108 different stimuli that were presented twice (a total of 216 stimuli in every block). Within the set of stimuli prepared for numerical or physical comparison, each digit and each physical size appeared on both sides of the visual field an equal number of times. The 108 stimuli included 36 congruent, 36 incongruent, and 36 neutral pairs of digits. A congruent stimulus was defined as a pair of digits in which a given digit was larger on both the relevant and irrelevant dimensions (e.g., 5 3). A neutral stimulus was defined as a pair of digits that differed only on the relevant dimension (e.g., 52 for the numerical comparisons or 55 for the physical comparisons). An incongruent stimulus was defined as a pair of digits in which a given digit was simultaneously larger on one dimension and smaller on the other (e.g., 52 ). The digits 2 through 8 were used. The two digits in each pair could be of the same physical size (in which case the pair served as a neutral for numerical comparisons) or could differ in height (physical dimension) by $1 \mathrm{~mm}$ (one digit was $6 \mathrm{~mm}$ and the other was $7 \mathrm{~mm}$ ) or by $2 \mathrm{~mm}$ (one digit was $7 \mathrm{~mm}$ and the other was $9 \mathrm{~mm}$ ). In addition, the two digits in each pair could be of the same numerical value (in which case the pair served as a neutral for physical comparisons) or could differ in numerical distance. There were three numerical distances: 1 (the digits 6-7, 4-5, and 2-3), 2 (the digits 6-8, 3-5, and 2-4), or 4 (the digits 4-8, 3-7, and 2-6). Accordingly, each distance included three different pairs of digits. In short, each block had 18 different possible conditions ( 2 physical sizes, 3 numerical distances, and 3 congruency conditions). Each condition had 12 trials for a total of 216 trials per block.

While the congruent and incongruent trials were the same for the two comparison tasks, the neutral stimuli were different for the two comparisons. Neutral stimuli in the physical comparison included the same digit in two different physical sizes. In order to keep the factorial design we created the neutral stimuli from 
the digits that were used for the other two conditions (congruent and incongruent). For example, because the pair 2-3 was used to produce congruent and incongruent stimuli for a numerical distance of 1 unit, neutral pairs created by using these two digits (i.e., 22 and 33 ) were included in the analysis as neutral trials for numerical distance 1. Similarly, because the pair 3-5 was used to produce the congruent and incongruent conditions for a numerical distance of 2 units, neutral pairs created by using these two digits (i.e., 33 and 5 5) were included in the analysis as neutral trials for numerical distance 2. Each digit from all three pairs of a given numeric distance was used to create four stimuli. For example, the digit 2 was presented in four stimuli: it was included twice in the analysis as a neutral trial for physical distance of $1 \mathrm{~mm}$ (e.g., 2-2: one digit of the pair was $6 \mathrm{~mm}$ and the other $7 \mathrm{~mm}$ ) and twice it was included in the analysis as a neutral trial for physical distance of $2 \mathrm{~mm}$ (e.g., 2 2: one digit of the pair was $7 \mathrm{~mm}$ and the other $9 \mathrm{~mm}$ ). In this way the comparison between the congruent, incongruent, and neutral conditions was made by using the same digits.

Neutral stimuli in the numerical comparison included two digits that were different in numerical value but of the same physical size. In order to keep the factorial design we created the neutral stimuli from the digits and physical sizes that were used for the other two conditions (congruent and incongruent). For example, the pair 2-3 was used to produce congruent and incongruent stimuli for a numerical distance of 1 unit. Hence, neutral pairs created by using these two digits (i.e., 23 ) were included in the analysis as neutral trials for a physical distance of 1 and $2 \mathrm{~mm}$. For a physical distance of 1 the two digits (2 3) were presented twice in a 6-mm height and twice in a 7 -mm height. For a physical distance of 2 , the two digits $\left(\begin{array}{l}2 \\ 2\end{array}\right)$ were presented twice in a 7-mm height and twice in a 9-mm height. Accordingly, the 7-mm stimuli were divided among the two conditions: half of them were stimuli for a distance of a $1 \mathrm{~mm}$ unit and half of them for a distance of a 2-mm unit. In this way the comparison between the congruent, incongruent, and neutral conditions was made using the same physical sizes.

Before every experimental block participants were presented with 36 practice trials. This block was similar to the experimental block with the following exceptions: (1) We used different Arabic numerals as stimuli. For numerical distances of 1 unit the digits were 3-4 and 5-6, for numerical distances of 2 units the digits were 4-6 and 5-7, and for numerical distances of 4 units the digits were 1-5 and 5-9. (2) Of the 48 possible congruent trials ( 2 physical sizes $\times 3$ numerical differences $\times 2$ stimuli per numerical distance $\times 2$ opposite sides of fixation $\times 2$ presentations) we randomly chose 12 trials. The same was done for the 48 possible incongruent and neutral trials.

Design. The variables manipulated were group (beginning of first grade, end of first grade, third grade, fifth grade, and university students), relevant dimension (physical vs numerical), physical distance (distance of $1 \mathrm{~mm}$ or $2 \mathrm{~mm}$ ), numerical distance (1,2, or 4) and congruity (incongruent, neutral, or congruent). Thus, we had a $5 \times 2 \times 2 \times 3 \times 3$ factorial design. Group was the only between-participants variable. 
Procedure. The participant's task was to decide which of two digits in a given display was larger. Each participant took part in one session composed of two different blocks. In one block "larger" was defined by physical size and in the other, it was defined by numerical value. The stimuli in each block were presented in a random order. Half of the participants performed physical comparisons first, and the other half performed numerical comparisons first. Before the experiment began, participants were given a practice block. The participants were asked to respond as quickly as possible but to avoid errors. They indicated their choices by pressing one of two keys corresponding to the side of the display with the chosen digit.

Each trial began with a fixation point presented for $300 \mathrm{~ms}$. Five hundred milliseconds after the fixation point was eliminated, a pair of digits appeared and remained in view until the participant pressed a key (but not for more than 5000 $\mathrm{ms}$ ). A new stimulus appeared $1500 \mathrm{~ms}$ after the participant's response.

\section{Results}

Error rates were generally low (at the beginning of first grade there were $6.8 \%$ errors, at the end of first grade there were $6.3 \%$, third grade had $2.7 \%$ errors, fifth grade had $3 \%$ errors, and university students had $2.5 \%$ errors) and therefore were not analyzed. For every participant in each condition the mean RT was calculated (for correct trials only). These means were subjected to a five-way analysis of variance (ANOVA) with group as the only between-subject factor and relevant dimension, physical distance, numerical distance, and congruity as within-subject factors.

All five main effects were significant. Speed of processing increased with age (mean RT for children at the beginning of first grade: $1375 \mathrm{~ms}$; end of first grade: $1150 \mathrm{~ms}$; third grade: $875 \mathrm{~ms}$; fifth grade: $773 \mathrm{~ms}$; and university students: 525 ms) $[F(4,75)=148, M S E=424,280, p<.001]$. Physical comparisons were made $257 \mathrm{~ms}$ faster than numerical comparisons $[F(1,75)=194.28, M S E=$ $266,851, p<.001]$. Participants responded faster to a large physical distance than to a small physical distance (mean RT for physical distance of $1 \mathrm{~mm}$ : $960 \mathrm{~ms}$; and for physical distance of $2 \mathrm{~mm}$ : $920 \mathrm{~ms})[F(1,75)=88.3, M S E=13,201, p<$ $.001]$. Similarly, participants responded faster to large numerical distances than to small ones (mean RT of numerical distance of 1 unit: 963 ms; 2 units: 940 ms; and 4 units: $917 \mathrm{~ms})[F(2,150)=48.5, M S E=10,159, p<.001]$. There was a significant congruity effect $[F(2,150)=147.1, M S E=34,751, p<.001]$ with mean RTs of 1013, 940, and $867 \mathrm{~ms}$ for incongruent, neutral, and congruent pairs, respectively.

The interaction between group and relevant dimension was significant $[F(4$, $75)=14.9, M S E=36,004, p<.001]$. Physical comparisons were always made faster than numerical comparisons, however, in separate comparisons of adjacent groups we found that the difference between physical and numerical judgments decreased as age increased. The difference in time between numerical and physical judgments was $128 \mathrm{~ms}$ smaller at the end of first grade compared with the 
beginning of first grade, $39 \mathrm{~ms}$ smaller in third grade compared with end of first grade, and $111 \mathrm{~ms}$ smaller in fifth grade compared with third grade $[F(1,75)=$ 19.19, $M S E=13,201, p<.001 ; F(1,75)=8.9, M S E=13,201, p<.01$; $F(1,75)=7.05, M S E=13,201, p<.01$, respectively]. No such significant difference was found between university students and fifth grade students.

The interaction between group, relevant dimension, and congruity was significant $[F(8,150)=2.9, M S E=37,210, p<.01]$ and is presented in Fig. 1. Since one of the main interests of this experiment was to study the development of automatic activation of numbers, we examined the simple interaction effects of congruity $\mathrm{X}$ group for each relevant dimension separately. The interaction between congruity and group was significant both when the relevant dimension was numerical $[F(8,150)=2.3, M S E=28,727, p<.05]$ and when it was physical $[F(8,150)=4.16, M S E=43,233, p<.001]$. Hence, within each relevant dimension, we computed the simple simple main effects of the congruent variable for the five groups. In the numerical comparison all simple simple main effects were significant [beginning of first grade: $F(2,30)=11.5, M S E=57,166, p<.001$; end of first grade: $F(2,30)=20.9, M S E=50,872, p<.001$; third grade: $F(2$, $30)=59.2, M S E=15,364, p<.001$; fifth grade: $F(2,30)=51.9, M S E=$

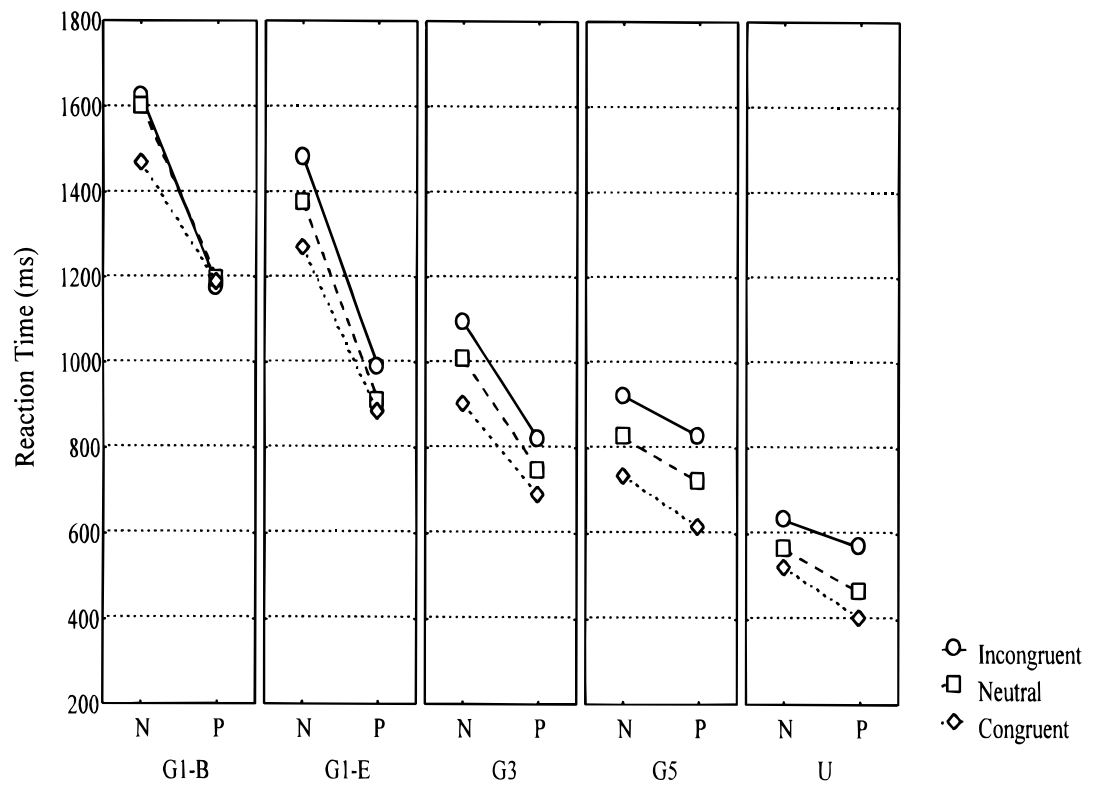

FIG. 1. The size congruity effect: mean reaction time as a function of group (grade), relevant dimension (task) and congruity. Each graph box belongs to a different age group, where $\mathrm{G}=$ grade; $\mathrm{B}=$ beginning of school year; $\mathrm{E}=$ end of school year; and $\mathrm{U}=$ undergraduate students. Relevant dimensions are $\mathrm{N}$, indicating numeric, and $\mathrm{P}$, indicating physical. 
$16,052, p<.001$; and university students: $F(2,30)=68.9, M S E=4,180, p<$ $.001]$. In the physical comparison all simple simple main effects of the congruity variable were significant except for the simple simple main effect in the beginning of first grade [end of first grade: $F(2,30)=22.6, M S E=12,726, p<.001$; third grade: $F(2,30)=8.5, M S E=49,501, p<.001$; fifth grade: $F(2,30)=$ 22.8, MSE $=47,425, p<.001$ and university students: $F(2,30)=26.3, M S E=$ $26,407, p<.001]$. We continued by analyzing the congruity variable within each relevant dimension.

Physical comparisons (numerical dimension is irrelevant). As can be seen in Fig. 1 (right side in each panel), the congruity effect starts to appear only at the end of the first grade $[F(1,75)=12.5, M S E=41,502, p<.001]$. At this time the size congruity effect (incongruent vs congruent) is composed mainly of an interference component (incongruent relative to neutral) $[F(1,75)=5.07, M S E=$ $58,629, p<.05]$. From the end of first grade until the third grade no significant change in the congruity effect is seen, nonetheless, a facilitatory component (congruent relative to neutral) appears in the third grade $[F(1,75)=5.95, M S E=$ $29,569, p<.01]$. Both facilitation and interference are shown by fifth grade $[F(1$, $75)=17.86, M S E=29,569, p<.001$ and $F(1,75)=9.48, M S E=58,629, p<$ .01 , respectively $]$ and by university students $[F(1,75)=3.6, M S E=29,569, p<$ .01 ; and $F(1,75)=9.25, M S E=58,629, p<.01$, respectively]. However, the size congruity effect is larger in the fifth grade $(233 \mathrm{~ms})$ compared with the third grade $(154 \mathrm{~ms})[F(1,65)=3.7, M S E=41,502, p<.05]$.

Numerical comparison (physical dimension is irrelevant). At the beginning of first grade the size congruity effect $[F(1,75)=28.6, M S E=39,385, p<.001]$ is composed mainly of a facilitatory component $[F(1,75)=36.4, M S E=22,485$, $p<.001]$. From the end of first grade an interference component appears also [size congruity effect at the end of first grade: $F(1,75)=53, M S E=39,385, p$ $<.001$; inhibitory component: $F(1,75)=22, M S E=24,312, p<.001$ and facilitatory component: $F(1,75)=23.4, M S E=22,485, p<.001]$.

The distance effect is indicated by the interaction of relevant dimension $\times$ numerical distance $[F(2,150)=33.7, M S E=13,458, p<.001]$ and is presented in Fig. 2. The numerical distance is significant only in the numerical block (when numerical dimension is relevant) $[F(1,75)=99.4, M S E=22,024, p<$ $.001]$ but not in the physical block. Group does not modulate this effect. The distance effect is one indication of automatic activation of a symbolic dimension, and therefore, may help in tracing the developmental changes in automatic processing of Arabic numerals. Thus, though the interaction with group was not significant $(F<1)$, the interaction between numerical distance and relevant dimension was tested separately for each group of participants. The distance effect was found to be significant in each one of the five groups: beginning of first grade $[F(2,30)=3.44, M S E=36,868, p<.05]$, end of first grade $[F(2,30)=8.15$, $M S E=12,455, p<.001]$, third grade $[F(2,30)=13.3, M S E=8,473, p<.001]$, fifth grade $[F(2,30)=9.8, M S E=7,182, p<.001]$, and university students $[F(2,30)=40.4, M S E=2,310, p<.001]$. 


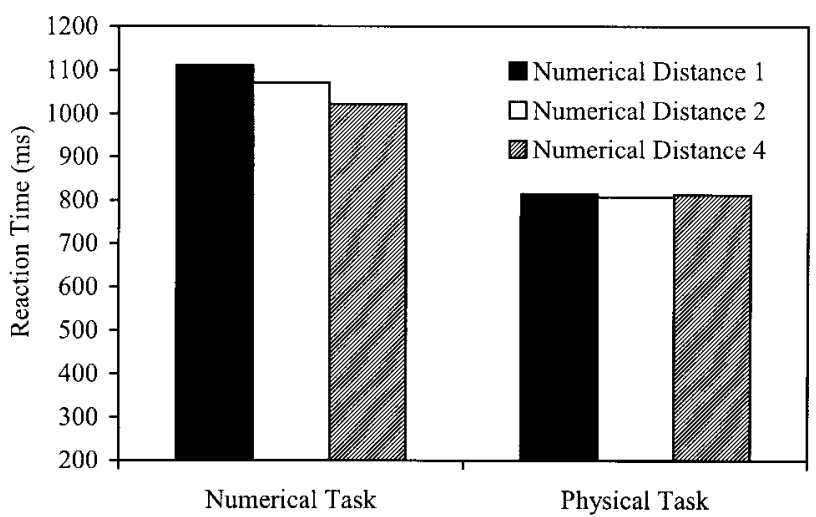

FIG. 2. The distance effect: mean reaction time as a function of relevant dimension (task) and numerical distance.

\section{Discussion}

Let us summarize the main results: (1) Speed of processing increases with age and the difference between RTs in physical and numerical tasks decreases with age. (2) When the physical dimension is relevant, a size congruity effect does not appear at the beginning of first grade. About 7 to 8 months later, numerically irrelevant information interferes with physical judgments. Thus, a size congruity effect starts to appear only at the end of first grade and is interference based. In the third grade a facilitatory component appears also. (3) A size congruity effect is found from the beginning of first grade, when the numerical dimension is relevant. (4) The numerical distance effect appears regardless of grade.

Size congruity, distance, and automaticity. We found an asymmetry between size congruity and distance effects at the beginning of first grade: There was a distance effect with no size congruity effect. These results support the two representations model. As mentioned in the introduction, this model suggests that young children, as well as adults, develop two different representations of numerical values. The first representation is an internal number line and is reflected in the distance effect. This representation can be accessed through an algorithmic process in an intentional autonomic fashion. First the digits are mapped into quantities on an analogical number line, which is used in turn to compare the digits. This comparison is conducted by a look-up process that probes the quantities on the number line. Since it is easier to compare quantities that are located far away from one another on this line than quantities that are located closer to one another on the line, there is a distance effect (Dehaene, 1989; Dehaene, Depoux, \& Mehler, 1990). The second representation is composed of instances of Arabic numerals. These instances enable one to classify numerals as "large" or "small." This representation can be accessed through a memory-based process of instance awakening in an autonomous automatic fashion. The comparative judgement decision differs 
for these two representations. We apply an algorithmic-based process (Dehaene, 1989; Dehaene, Depoux, \& Mehler, 1990) to the number scale and a memory retrieval process (Logan, 1988) to the memory-based representation. There is the ability to access each one of the two representations independently.

At the beginning of first grade, children are perfectly able to make a "large" vs "small" classification of quantities and objects, but they have not yet accumulated many instances of comparisons between numerical values of Arabic digits. Hence, their ability to judge which of two Arabic digits is the larger one cannot be retrieved from memory in a single step (i.e., in an autonomous automatic fashion) (Logan, 1988). Therefore, the irrelevant numerical value had no effect, so that size congruity did not appear in the physical task at the beginning of first grade. In contrast, during their first 6 years of life, young children develop the algorithm-based process, which exploits the internal scale (Gallistel \& Gelman, 1992; Siegler, 1986; Sophian, 1998; Sophian, Garyantes, \& Chang, 1997; Strauss \& Curtis, 1981; Wynn, 1992, 1995). This algorithm-based process is reflected in the distance effect (beginning of first grade) (see also Duncan \& McFarland, 1980; HuntleyFenner \& Cannon, 2000; Sekular \& Mierkiewicz, 1977; Temple \& Posner, 1998; Xu \& Spelke, 2000). It is important to note that the distance effect appears only when numerical values are relevant and not when they are irrelevant (physical judgements). This can be explained by Logan's (1988) assumption that in the race between memory retrieval and algorithm based processes, the former usually wins. However, when the task requires attending to the numerical dimension, participants use the algorithmic process to retrieve the required information from the number line. As we suggested earlier, this algorithmic process is done in an intentional automatic fashion (Tzelgov, Henik, Sneg, \& Baruch, 1996) and is reflected in the distance effect in the numerical comparisons.

Young children are slower to respond than older children. What accounts for this difference? One reason could be that the association of size (physical or numerical) with an arbitrary key-press is much more difficult for younger children (see Kail, 1991). However, despite these slower responses, there is a bigger difference between the slow numerical processing and the fast physical processing in young children than in older children. Hence, at an early age, the ability to confront a physical dimension is very different from the ability to confront a numerical dimension and it may rely on memory retrieval of instances (assuming that instances of physical comparisons accumulate earlier) (Yonas, Granrud, \& Pettersen, 1985). As age increases instances of comparisons of numerical values of Arabic digits begin to accumulate and therefore are processed much like physical sizes. Considering the fact that physical sizes are automatically processed from infancy (Yonas, Granrud, \& Pettersen, 1985), we argue that as age increases, numerical values of Arabic digits are being processed faster and in an autonomous automatic fashion, similar to the processing of physical sizes.

Furthermore, several reports have suggested dissociation between the interference and the facilitatory components of the Stroop effect (Henik, Singh, Beckley, \& Rafal, 1993; Lindsay \& Jacoby, 1994; Posner, 1978; Tzelgov, Henik, \& Berger, 
1992). The facilitatory component is supposed to involve processes that are more automatic since they are less subjected to strategic control (for example, see Tzelgov, Henik, \& Berger, 1992). Accordingly, our results show that at the end of first grade, size congruity in the physical task (when the numerical dimension was irrelevant) was composed of an interference component, and only in third grade the facilitation component appeared also. Hence, the ability to categorized Arabic numerals as "large" or "small" develops with age and becomes automatic at a more advanced age. As suggested earlier, the automaticity of classifying digits as "large" vs "small" depends on the accumulation of instances in memory so that such classification can be retrieved from memory in a single step (Logan, 1988). Note that in the numerical task (when the physical dimension was irrelevant), size congruity was composed only of a facilitation component at the beginning of first grade. As mentioned earlier, physical sizes are automatically processed from a very early age (Yonas, Granrud, \& Pettersen, 1985), therefore the facilitation component, which is more automatic in nature, appeared earlier than the facilitation component in the physical task (when numerical values were irrelevant).

As seen under Results, the numerical comparisons were significantly slower than the physical comparisons. It might be argued that there is a memory-based process from the beginning of first grade in which classification of "large" vs "small" of numerical values is done. In this case access to these representations might not be completed in time to interfere with the physical comparison. We believe that our results support the idea that numerical processing at the beginning of first grade is not yet automatic, at least not for the crude classifications of magnitudes into "large" or "small" (which is memory based). Hasher and Zacks (1979) and Logan (1988) argued that the degree of automaticity is reflected in the speed of processing and correlates with proficiency; as skill develops processing becomes faster and might be produced in a single step. Logan (1988) suggested that the memory-based mechanism develop with practice. Since we found that the speed of processing increases with age, we argue, according to Hasher and Zacks (1979) and Logan (1988), that in a more advanced age and with school practice, the ability to classify Arabic digits into "large" or "small" becomes autonomous automatic and those instances can be retrieved from memory in a single step.

Maturation or schooling. One may argue that the significant improvement in number processing in first grade may be due to maturational factors. This idea is consistent with the slow maturation of frontal areas in the brain, which reach full development only at the age of 7 years old (Diamond, 1992; Thatcher, 1991). At this age, the participants in our experiment were at the end of first grade, when size congruity started to appear. The design of our study does not allow us to separate maturing and schooling effects. However, the argument that changes in children's numerical knowledge are influenced only by maturation is inconsistent with two other findings. First, there was a marginally significant difference in the size congruity effect between third and fifth grade participants. Mean ages in these groups were 8.8 and 11 years old in the third and fifth grades, respectively. By this time in life, brain areas that are related to size comparisons have reached 
their full development. Temple and Posner (1998), for example, found that the brain area responsible for abstract representation of Arabic numerals and arrays of dots is located in the inferior parietal area of the brain. This area develops and is responsive to number comparison before 5 years of age and before formal mathematical training. Second, the fact that a distance effect but not a size congruity effect was found in all ages might imply that schooling improves the ability to access the internal magnitudes, at least to those symbols that indicate large or small quantities. The subcomponents of skilled performance, which become automatic with practice, are supposed to serve the skilled performer by being executed effortlessly and faster (Hasher \& Zacks, 1979; Shiffrin \& Schneider, 1977). Accordingly, the degree of skill acquired during learning at school might have influenced the speed taken to classify the Arabic digits into a "large" vs "small" scale. Thus, the distance effect but not the size congruity effect appeared in young children who were not highly skilled with the use of Arabic digits.

Moreover, Griffin, Case, and Capodilupo (1995) have shown that children who lack central numerical concepts did not succeed in formal mathematical instruction. After these concepts had been taught these children developed an internal representation of magnitude and, thus, performed as well as children who had firm numerical concepts. This indicates that numerical knowledge may be influenced by schooling and not only by maturing.

The study of Girelli et al. (2000). Our results are similar to the results found in the experiments of Girelli, Lucangeli, and Butterworth (2000) (see also Butterworth, 1999). Both studies found a size congruity effect in the earliest age group, that is, beginning of first grade in our experiment and 4-year-old children in Butterworth's (1999) experiment in the numerical task (when the physical dimension was irrelevant). However, Girelli, Lucangeli, and Butterworth (2000) did not find a size congruity effect in the first grade group in the physical task (when the numerical dimension was irrelevant). Since we divided the first grade children into two groups, that is, beginning and end of first grade, we were able to find that a size congruity effect starts to appear at the end of first grade, earlier than argued by Girelli, Lucangeli, and Butterworth. In addition, in contrast with Girelli, Lucangeli, and Butterworth, we used three different numerical distances, that is, numerical distances of 1,2 , or 4 . The use of three numerical distances enables one to examine the idea that distance has a monotonic relationship with responding. Had we used only two distances we would not have been able to rule out nonmonotonic relations. Hence, using three numerical distances enabled us to study developmental changes in the distance effect rather than only the laterality effect.

It should be noted that Girelli, Lucangeli, and Butterworth (2000) examined the effect of laterality by comparing bilateral pairs (i.e., one digit was smaller and the other larger than 5) and unilateral pairs (i.e., both digits either smaller or larger than 5). For the bilateral stimuli they employed pairs with distance 5 (e.g., 16 ), while for the unilateral stimuli they employed pairs with distance 1 (e.g., 12 ). Thus, it is possible to examine effects of distance by comparing these two conditions. The authors did report a significant effect of laterality (i.e., RTs to bilater- 
al pairs were shorter than RTs to unilateral pairs) in numerical comparisons, regardless of age. However, this arrangement confounds laterality and distance, so the fact that bilateral pairs were responded to faster than unilateral pairs may be due either to the influence of laterality or to the distance effect. Our design included three numerical distances: 1 and 2 for unilateral pairs (6-7, 4-5, and 2-3 and 6-8, 3-5, and 2-4) and 4 for bilateral pairs (4-8, 3-7, and 2-6). In order to examine effects of distance that are not confounded by laterality, we compared only unilateral pairs with distances 1 and 2 . The results of these analyses support the conclusions presented previously. Examination of the numerical task showed a significant distance effect regardless of group $[F(1,75)=10.21, M S E=$ $12,164.8, p<.05]$. Despite the lack of interaction with group we compared responses to these two distances in the youngest group of participants (i.e., beginning of first grade). Within this group RTs to distance 1 (1603 ms) were significantly slower than RTs to distance $2(1570 \mathrm{~ms})[F(1,75)=8.2, M S E=17,839$, $p<.05]$. This suggests that at the beginning of first grade children do show the distance effect, independent of any laterality effect.

\section{CONCLUSIONS}

To summarize, we presented a model of internal representation of numbers according to which there are two different internal representations of magnitude (the number scale and the instance of pairs of digits) which are retrieved by different processes.

We can conclude by saying that our results help understand the development of automatic processing of numerical information. The ability to understand and automatically process the quantitative values of Arabic numerals may play an important role in the acquisition and implementation of skilled calculation (Griffin, Case, \& Capodilupo, 1995). Understanding the development of these abilities may have profound implications for theories of education and child development in the field of number processing.

\section{REFERENCES}

Anderson, J. R. (1993). Rules of the mind. Hillsdale, NJ: Erlbaum.

Banks, W. P. (1977). Encoding and processing of symbolic information in comparative judgments. In G. H. Bower (Ed.), The psychology of learning and motivation (pp. 101-159). San Diego, CA: Academic Press.

Banks, W. P., Mermelstein, R., \& Yu, K. H. (1982). Discrimination among perceptual and symbolic stimuli. Memory \& Cognition, 10, 265-275.

Besner, D., \& Coltheart, M. (1979). Ideographic and alphabetic processing in skilled reading of English. Neuropsychologia, 17, 467-472.

Boucart, M., \& Humphteys, G. W. (1994). Attention to orientation, size, luminance, and color: Attentional failure within the form domain. Journal of Experimental Psychology: Human Perception and Performance, 20, 61-80.

Butterworth, B. (1999). The mathematical brain. London: Macmillan.

Carr, T. H. (1992). Atomaticity and cognitive anatomy: Is word recognition automatic. American Journal of Psychology, 105, 201-237.

Dehaene, S. (1989). The psychophysics of numerical comparison: A reexamination of apparently incompatible data. Perception \& Psychophysics, 45, 557-566. 
Dehaene, S. (1992). Varieties of numerical abilities. Cognition, 44, 1-42.

Dehaene, S. (1997). Our numerical heritage: The adult number line. In S. Dehaene (Ed.), The number sense (pp. 64-90). Oxford, UK: Oxford Univ. Press.

Dehaene, S., \& Akhavein, R. (1995). Attention, automaticity and levels of representation in number processing. Journal of Experimental Psychology: Learning, Memory and Cognition, 21, 314-326.

Dehaene, S., \& Changeux, J. (1993). Development of elementary numerical abilities: A neuronal model. Journal of Cognitive Neuroscience, 5, 390-406.

Dehaene, S., Dupoux, E., \& Mehler, J. (1990). Is numerical comparison digital: Analogical and symbolic effect in two-digit number comparison. Journal of Experimental Psychology: Human Perception and Performance, 16, 626-641.

Diamond, A. (1992). In H. S. Levin \& H. M. Eisenberg (Eds.), Frontal lobe function and dysfunction. New York: Oxford Univ. Press.

Duncan, E. M., \& McFarland, C. E. (1980). Isolating the effect of symbolic distance and semantic congruity in comparative judgments: An additive-factors analysis. Memory \& Cognition, 2, 95-110.

Gallistel, C. R., \& Gelman, R. (1992). Preverbal and verbal counting and computation. Cognition, 44, 43-74.

Gelman, R. (1978). Counting in the preschooler: What does and does not develop. In R. S. Siegler (Ed.), Children's thinking: What develops? (pp. 213-241). Hillsdale, NJ: Erlbaum.

Gelman, R. (1982). Accessing one-to-one correspondence: Still another paper about conservation. British Journal of Psychology, 73, 209-220.

Gelman, R. (1994). Constructivism and supporting environments. In D. Tirosh (Ed.), Human development. Vol. 6: Implicit and explicit knowledge: An educational approach (pp. 55-82). Norwood, NJ: Ablex.

Girelli, L., Lucangeli, D., \& Butterworth, B. (2000). The development of automaticity in accessing number magnitude. Journal of Experimental Child Psychology, 76, 104-122.

Griffin, S., Case, R., \& Capodilupo, A. (1995). Teaching for understanding: the importance of central conceptual structure in the elementary mathematics curriculum. In A. McKeough, J. Lupart, \& A. Marini (Eds.), Teaching for transfer: Fostering generalization in learning (pp. 123-151). Mahwah, NJ: Erlbaum.

Hasher, L., \& Zacks, R. T. (1979). Automatic and effortful processes in memory. Journal of Experimental Psychology: General, 108, 356-388.

Henik, A., Singh, J., Beckley, D. J., \& Rafal, R. D. (1993). Disinhibition of mental word reading in Parkinson's disease. Cortex, 29, 589-599.

Henik, A., \& Tzelgov, J. (1982). Is three greater than five: The relation between physical and semantic size in comparison tasks. Memory \& Cognition, 10, 389-395.

Huntley-Fenner, G., \& Cannon, E. (2000). Preschooler's magnitude comparisons are mediated by a preverbal analog mechanism. Psychological Science, 11, 147-152.

Kail, R. (1991). Developmental change in speed of processing during childhood and adolescence. Psychological Bulletin, 109, 490-501.

Lindsay D. S., \& Jacoby, L. L. (1994). Stroop process dissociations: The relationship between facilitation and interference. Journal of Experimental Psychology: Human Perception and Performance, 20, 219-234.

Link, S. (1990). Modeling imageless thought: The relative judgment theory of numerical comparison. Journal of Mathematical Psychology, 34, 2-41.

Logan, G. D. (1985). Skill and automaticity: Relations, implications and future directions. Canadian Journal of Psychology, 39, 367-386.

Logan, G. D. (1988). Toward an instance theory of automatization. Psychological Review, 95, 492-527. Moyer, R. S. (1973). Comparing objects in memory: Evidence suggesting an internal psychology. Perception \& Psychology, 13, 180-184.

Moyer, R. S., \& Bayer, R. H. (1976). Mental comparison and the symbolic distance effect. Cognitive Psychology, 8, 228-246.

Moyer, R. S., \& Landauer, T. K. (1967). The time required for judgments of numerical inequality. Nature, 215, 1519-1520. 
Paivio, A. (1975). Perceptual comparisons through the mind's eye. Memory \& Cognition, 3, 635-647.

Parkman, J. M. (1971). Temporal aspects of digit and letter inequality judgments. Journal of Experimental Psychology, 91, 191-205.

Piaget, J. (1952). The child's conception of number. New York: Norton.

Piaget, J. (1953). How children form mathematical concepts. Scientific American, 189, 74-79.

Posner, M. I. (1978). Chronometric exploration of mind. Hillsdale, NJ: Erlbaum.

Rabinowitz, F. M., Grant, M. J., Howe, M. L., \& Walsh, C. (1994). Reasoning in middle childhood: A dynamic model of performance on transitivity tasks. Journal of Experimental Child Psychology, 58, 252-288.

Schwarz, W., \& Heinze, H. J. (1998). On the interaction of numerical and size information in digit comparison: A behavioral and event-related potential study. Neuropsychologia, 36, 1167-1179.

Sekular, R., \& Mierkiewicz, D. (1977). Children's judgment of numerical inequality. Child Development, 48, 630-633.

Shifrin, R. M., \& Schneider, W. (1977). Controlled and automatic human information processing: Perceptual learning, automatic attending and general theory. Psychological Review, 84, 127-190.

Siegler, R. S. (1986). Unities across domains in children's strategy choices. In M. Perlmutter (Ed.), Perspectives for intellectual development. Vol. 19: The Minnesota Symposia Child Psychology (pp. 1-48). Hillsdale, NJ: Erlbaum.

Sophian, C. (1998). Limitation on preschool children's knowledge about counting: Using counting to compare two sets. Developmental Psychology, 24, 634-640.

Sophian, C., Garyantes, D., \& Chang, C. (1997). When three is less than two: Early developments in children's understanding of fractional quantities, Developmental Psychology, 3, 731-744.

Strauss, M. S., \& Curtis, L. E. (1981). Infants perception of numerosity. Child Development, 52, $1146-1152$.

Ta'ir, J., Brezner, A., \& Ariel, R. (1997). Profound developmental dyscalculia: Evidence for a cardinal/ordinal skills acquisition device. Brain and Cognition, 35, 184-206.

Temple, E., \& Posner, M. I. (1998). Brain mechanisms of quantity are similar in 5-year-olds and adults. Proceedings of the National Academy of Sciences USA, 95, 7836-7841.

Thatcher R. W. (1991). Maturation of the human frontal lobes: Psychological evidence for staging. Developmental Neuropsychology, 7, 397-419.

Tzelgov, J., Henik, A., \& Berger, J. (1992). Controlling Stroop effects by manipulating expectations for color words. Memory \& Cognition, 20, 727-735.

Tzelgov, J., Henik, A., Sneg, R., \& Baruch, O. (1996). Unintentional reading via the phonological route: The Stroop effect with cross-script homophones. Journal of Experimental Psychology: Learning, Memory and Cognition, 22, 336-349.

Tzelgov, J., Meyer, J., \& Henik, A. (1992). Automatic and intentional processing of numerical information. Journal of Experimental Psychology: Learning, Memory \& Cognition, 18, 166-179.

Tzelgov, J., Yehene, V., Kotler, L., \& Alon, A. (2000). Automatic comparisons of artificial digits never compared: Learning linear ordering relations. Journal of Experimental Psychology: Learning, Memory and Cognition, 26, 1-18.

Whalen, J., Gallistel, W. J., \& Gelman, R. (1999). Nonverbal counting in humans: The psychophysics of number representation. Psychological Science, 10, 130-137.

Wynn, K. (1992). Addition and subtraction by human infants. Nature, 358, 749-750.

Wynn, K. (1995). Origins of numerical knowledge. Mathematical Cognition, 1, 35-90.

Xu, F., \& Spelke, E. S. (2000). Large number discrimination in 6 month old infants. Cognition, 74, B1-B11.

Yonas, A., Granrud, C. E., \& Pettersen, L. (1985). Infants' sensitivity to relative size information for distance. Developmental Psychology, 21, 161-167.

Zbrodoff, N. J., \& Logan, G. D. (1986). On the autonomy of mental process: A case study of arithmetic. Journal of Experimental Psychology: General, 115, 118-130.

Received September 15, 2000; revised July 2, 2001 